\title{
The retrospective experience of day-surgery semi tubeless ultra-mini percutaneous nephrolithotomy
}

\author{
Qi Chen", Yang Cao ${ }^{\#}$, Lei Xia, Hai Zhong, Zhebin Du, Hanqing Xuan, Mujun Lu^ \\ Department of Urology, Renji Hospital, School of Medicine, Shanghai Jiao Tong University, Shanghai, China \\ Contributions: (I) Conception and design: M Lu, Q Chen; (II) Administrative support: M Lu; (III) Provision of study material: Q Chen, Y Cao, L \\ Xia; (IV) Collection and assembly of data: Q Chen, H Zhong, Z Du, H Xuan; (V) Data analysis and interpretation: Q Chen, Y Cao; (VI) Manuscript \\ writing: All authors; (VII) Final approval of manuscript: All authors. \\ \#These authors contributed equally to this work. \\ Correspondence to: Mujun Lu. Department of Urology, Renji Hospital, School of Medicine, Shanghai Jiao Tong University, Shanghai 200127, China. \\ Email: lumujun@163.com.
}

\begin{abstract}
Background: To retrospectively assess the experience of day-surgery semi tubeless ultra-mini percutaneous nephrolithotomy (UMP) for the treatment of kidney stones by experienced surgeons.

Methods: Clinical data of 358 patients with kidney stones (254 males and 104 females; mean age: $59.60 \pm 11.70$ ) who were performed UMP surgery in Shanghai Jiao Tong University School of Medicine affiliated Renji Hospital from June 2015 to December 2018. Patient demographics, operative data, complications, and readmission rates were recorded. Day-surgery UMP was defined as discharge of patients either the same day or within $24 \mathrm{~h}$ after surgery. Semi tubeless UMP was defined as no placement of DJ and nephrostomy tube after surgery.

Results: The average size of stones was $14.56 \pm 6.24 \mathrm{~mm}$ (range, 4-30 mm). There are solitary stones in 192 cases, multiple stones in 142 cases and 29 cases with Staghorn stones. F13 outer sheath was used for all operations; 358 patients completed UMP on the day of admission; 326 (91.06\%) patients achieved sameday discharge or received overnight observation prior to discharge, and 32 patients $(8.94 \%)$ required full admission (longer than $24 \mathrm{~h}$ ). The readmission rate was $0.56 \%$ (2 patients). The postoperative complications within 1 week occurred in $36(10.06 \%)$ patients, including 23, 10, 3 of grades I, II, IIIa complications (Clavien-System). The average operation time was $29.64 \mathrm{~min}$ and the hemoglobin drop were $13.42 \pm 9.55 \mathrm{~g} / \mathrm{L}$. The stone clearance rate was $91.62 \%$ (328/358). The semi tubeless rate war 95.25\% (341/358).
\end{abstract}

Conclusions: For day-surgery semi tubeless UMP, experienced surgeons gain excellent patient outcomes in appropriately selected patients. Day-surgery semi tubeless UMP is worth promoting.

Keywords: Ultra-mini percutaneous nephrolithotomy (UMP); day-surgery; kidney stones; semi-tubeless

Submitted Jul 03, 2020. Accepted for publication Nov 24, 2020.

doi: $10.21037 /$ tau-20-1060

View this article at: http://dx.doi.org/10.21037/tau-20-1060

\section{Introduction}

On a global basis, the incidence of kidney stones is gradually increasing in different races, ages, or sexes (1). Since percutaneous nephrolithotomy (PCNL) with the standard channel was first introduced by Fernström and Johansson in 1976, it became the main modality for $>2 \mathrm{~cm}$ stones and traditionally was performed on an inpatient basis (2). As technology advances, surgeons seek more

^ ORCID: Yang Cao, 0000-0002-5023-680X; Mujun Lu, 0000-0002-3404-1260. 
minimally invasive, more effective, and more economical methods. All these technical inventions are based on the theory: the occurrence rate of PNL complications is closely associated with the size of the channel, and the smaller the channel is, the lower the risk of complications is (3-5). For this reason, in 2013, Desai et al. (6) introduced the ultra-mini-PNL (UMP), in which a $6 \mathrm{Fr}$ mini nephroscope is used through a 11/13 Fr metal sheath and stones are fragmented with a laser. At present, the size standard for UMP treatment of kidney stones has not been clearly defined, and it is generally considered to be between $1-2 \mathrm{~cm}$. This mainly considers that complications are related to operation time. But for experienced surgeons, 2$3 \mathrm{~cm}$ stones can also be done well. Compared with traditional PCNL, UMP has the following characteristics: visualization before dilation, smaller channel (F11/13), lower trauma and more possibilities of tubeless. Because of these advantages, we have been starting the day-surgery semi tubeless UMP since the June 2015. In addition to the meaning of not indwelling the DJ tube and nephrostomy tube, semitubeless means that the ureteral catheter used during the operation is temporarily retained and fixed with the urethral catheter after surgery, which are removed together when discharged from the hospital the next day after surgery. The potential benefits of this management strategy include improved patient recuperation, cost savings, and superior utilization of hospital resources (7). However, even with these advances and advantages, day-surgery semi tubeless UMP is not appropriate for every patient. The protocol of the selection criteria was developed jointly by hospital managers, surgeons, anesthesiologists. Herein, we evaluated the safety and efficacy of day-surgery semi tubeless UMP in appropriately selected patients using a systematic protocol. All procedures were performed by experienced urologists, each of whom had completed more than 1,000 PCNL operations. We present the following article in accordance with the STROBE reporting checklist (available at: http:// dx.doi.org/10.21037/tau-20-1060).

\section{Methods}

\section{Clinical data}

We reviewed 358 patients with kidney stones [254 males and 104 females; mean age: 59.60 \pm 11.70 (range, 22-83 years)] who received surgery in Department of Urology, Renji Hospital Affiliated to Shanghai Jiao Tong University School of Medicine from June 2015 to
December 2018. All procedures performed in this study were in accordance with the Declaration of Helsinki (as revised in 2013). This study was reviewed and approved by Ethics Committee of Renji Hospital Affiliated to Shanghai Jiao Tong University School of Medicine (KY2020-058). All patients enrolled completed the informed consent form.

Since 2010, ureteroscope lithotripsy (URSL) and flexible ureteroscope lithotripsy (FURSL) have become day surgery in our hospital. A protocol for day-surgery semi tubeless UMP was conceived from the past experience and required multifaceted coordination. All examinations and evaluations were performed in the urology clinic and anesthesia clinic. For anesthesiologists, patients need to meet the following conditions: ASA grade $\leq 2$; WHO performance status $\leq 2$; no coronary heart disease (CHD). Although in the selection criteria, the age is not allowed more than 75 years. But, in this study, two patients were over 75 years old: one was 83 and the other was 76 . The two patients met the inclusion criteria except for age. They had a strong demand for day surgery. In the selection criteria of the stone size, the maximum diameter did not exceed $3 \mathrm{~cm}$. All patients had no UTI and stopped taking anticoagulants for at least two weeks before surgery. Additional examinations included collection of the medical history, physical examination, blood and urine test, bleeding and coagulation series, hepatic and renal function, blood electrolytes, indicators of UTI, B-ultrasound of urinary system and CTU. The baseline characteristics of patients were shown in Table 1 . Day-surgery was defined as discharge of patients either the same day or within $24 \mathrm{~h}$ after surgery (8).

\section{Surgical equipment and procedures}

A 13F ultra-mini percutaneous nephroscope set from LUT GmbH (Leben und Technologie, Germany) was used for the surgery, and it was composed of a $3.5 \mathrm{~F}$ ultra-mini nephroscope, a $7.5 \mathrm{~F}$ operating frame, and a $13 \mathrm{~F}$ sheath and obturator equipped with guide wire duct. After the patients adopted the lithotomy position under general anesthesia, cystoscopic retrograde indwelling of a F6 ureteral catheter was performed through the urethra. Following that, the prone position was taken, and under type-B ultrasonic real-time location, a $16 \mathrm{G}$ puncture needle was used to puncture the targeted calyx, and the metal guide wire with J-type head and hard-straight body was placed. A $4 \mathrm{~cm}$ skin incision was made, and F10 and F14 fascia dilator was used in turn to dilate the passage until F14 along the guide wire, and a F13 UMP sheath was pushed in along the guide 
Table 1 Baseline characteristics of patients

\begin{tabular}{ll}
\hline Patient characteristics & Results \\
\hline Age (yeas, mean \pm SD) & $59.60 \pm 11.70$ \\
Gender (male/female) & $254(70.95 \%) / 104(29.05 \%)$ \\
Laterality (left/right) & $206(56.75 \%) / 157(43.25 \%)$ \\
Stone diameter (mm, mean \pm SD) & $14.56 \pm 6.24$ \\
Stone CT Hounsfield unit & $1,124.9 \pm 298.1$ \\
Stone type & \\
Single & 192 \\
Multiple & 142 \\
Staghorn & 29 \\
\hline
\end{tabular}

wire. Finally, the collective system structure was observed to look for the stones. Lumines holmium laser was adopted to perform lithotripsy, during which the power could be changed according to the stone size, location and physical properties in the operation process (energy fluctuation range: $0.8-2.0 \mathrm{~J}$; frequency range, $10-25 \mathrm{~Hz}$ ). During the operation, the stones were crushed to $<3 \mathrm{~mm}$ in size, and with the help of the vortex formed by artificial water flow from the retrograde ureteral catheter combined with the wash pipe on inner sheath wall on the front-end of the endoscope body, the stone fragments were directly washed out via the endoscopic sheath. After confirming that there was no obvious residual stone under ultrasound combined with endoscopic observation, semi tubeless procedures were performed routinely after surgery while the ureteral catheter was temporarily retained. The stone samples were submitted for infrared spectroscopy to analyze their compositions. First day after surgery, CT scan of kidney and ureter was performed to evaluate whether there was residual stone and perirenal hematoma. Meanwhile, the biochemical examinations were completed. If there was no fever, obvious hematuria, wound hemorrhage, urinary extravasation, or septicopyemia, and the anal exsufflation function was recovered, the urinary catheter and ureteral catheter could be removed. In this study, residual stone was defined as presence of upper urinary tract stones with the size $>3 \mathrm{~mm}$ after surgery.

\section{Interested parameters}

Statistical outcomes were day-surgery success rate, semi tubeless rate, stone-free rate (SFR), readmission rate and complications, which were graded according to the Clavien classification. Other relevant data were also included in the statistics: operative time, postoperative hemoglobin changes (hemorrhage) and changes of the renal function. All outcomes can well determine the safety and effectiveness of day-surgery semi tubeless UMP. The available CT scan were performed the first day after surgery. Stone free status was also determined and defined both as a $\leq 3 \mathrm{~mm}$ residual fragment and as no residual stone burden (9). All count data were expressed in the form of mean \pm standard deviation (SD).

\section{Statistical analysis}

Data were collated using Microsoft Excel and analysed with SPSS version 16.0. Continuous data were recorded as the mean \pm SD.

\section{Results}

A total of 358 patients underwent planned day-surgery semi tubeless UMP. There were 104 females (29.05\%) and 254 males $(70.95 \%)$, with an overall mean age of $59.60 \pm 11.70$ years. Mean stone size was $14.56 \pm 6.24 \mathrm{~mm}$ (range, 4-30 mm). There were 190 cases of single stone, 139 cases of multiple stones ( 3 patients with multiple stones of both sides), and 29 cases of staghorn stones. All patients can only undergo day surgery if urine culture is negative in outpatient (Table 2).

All patients $(\mathrm{n}=358)$ were performed UMP with tract size $13 \mathrm{~F}$ on the day of admission. $326(91.06 \%)$ patients which received overnight observation were discharged within 24 hours from admission; 32 patients (8.94\%) required full admission (longer than $24 \mathrm{~h}$ ). DJ tubes were placed in 11 patients after surgery and nephrostomy tubes were 6 . The semi tubeless rate war $95.25 \%$ (341/358). The reasons for placement are: large intrapelvic clot (3 cases); PUJ stenosis (3 cases); caliceal diverticulum stones (2 cases); 9 cases of diabetics with long operation time ( $>45 \mathrm{~min})$. The rest retained the ureteral catheters which were removed together with the urinary catheter on the first postoperative day. Of the 32 patients who were not discharged on time, 7 cases were due to fever exceeding $38.5^{\circ} \mathrm{C}$ after surgery; 10 cases of large perirenal hematoma $(>3 \mathrm{~cm})$ detected by CT scan; 9 cases because of subjective postoperative pain; 2 cases of hypokalemia; 4 of obvious hematuria. It should be noted that no patient had urosepsis.

The postoperative complications within 1 week after 
Table 2 Intraoperative and postoperative variables

\begin{tabular}{lc}
\hline Variables & Results \\
\hline Intraoperative blood loss (g/L, mean \pm SD) & $13.42 \pm 9.55$ \\
Rate of perioperative complications \% & 10.06 \\
Delayed discharge rate \% & 8.94 \\
$\begin{array}{l}\text { Day-surgery rate \% (discharge in the next day } \\
\text { morning) }\end{array}$ & 91.06 \\
$\begin{array}{l}\text { Readmission rate \% } \\
\begin{array}{l}\text { Stone-free rate } \leq 3 \text { mm or no residual \% (CT } \\
\text { on the first day after surgery) }\end{array}\end{array}$ \\
$\begin{array}{l}\text { Semi tubeless rate \% (neither DJ stent nor } \\
\text { nephrostomy tube) }\end{array}$ & 9.56 \\
\hline
\end{tabular}

Table 3 Postoperative complications of day-surgery semi tubeless UMP

\begin{tabular}{lll}
\hline Clavien grade & Total & Complications \\
\hline Grade I & 15 & $\begin{array}{l}\text { Hypokalemia }(n=2) \\
\text { Perirenal hematoma }(n=9) \\
\text { Obvious hematuria }(n=4)\end{array}$ \\
Grade II & 10 & Fever $\left(\geq 38.5^{\circ} \mathrm{C}\right)$ \\
Grade IIla & 3 & $\begin{array}{l}\text { Renal embolization, one got } \\
\text { transfusion, one with perirenal } \\
\text { hematoma }\end{array}$ \\
\hline
\end{tabular}

UMP, ultra-mini percutaneous nephrolithotomy.

UMP occurred in $28(7.82 \%)$ patients, including 15,10 , 3 of grades I, II, IIIa complications (Clavien-System) (Table 3). Four patients underwent obvious hematuria and were discharged after hemostasis using ethamsylate. Hypokalemia in 2 cases, who received potassium supplementation after surgery. There were 10 cases of fever $\geq 38.5^{\circ} \mathrm{C}$. Seven of them in 8 hours after surgery, and 3 of them developed less than a week after discharge. All recovered after treatment by indomethacin and antibiotics. Except for one patient with embolization on the third day after operation, hematomas were absorbed during the second month of postoperative review in the remaining 9 patients with perirenal hematoma. There were no cases of urosepsis before or after surgery. Three patients got renal embolized postoperatively. One patient was embolized on the third day after operation, and the other two patients were readmitted to the hospital due to severe hematuria, and were embolized on the fifth and seventh day after surgery.
The readmission rate for day-surgery patients was $0.56 \%$ and one of them required blood transfusion. Angiographic embolization was successful in stopping bleeding. No other major complications, including pneumothorax, ureteral injury and visceral damage.

According to the definition of residual stone above $3 \mathrm{~mm}$, the CT scan on the first day after the operation showed that the SFR was $91.62 \%(328 / 358)$ and the CT scan after one month after the operation showed that the SFR was 94.69\% (339/358).

Figure 1 shows the results of stone analysis, which were obtained using infrared spectroscopy. Most stones were mixed ingredients. The closer to the front, the higher the proportion.

\section{Discussion}

Walid Shahrour and Sero Andonian et al. reported their initial experience of day-surgery tubeless PCNL performed on 10 patients. They commented that day-surgery PCNL is safe and feasible in highly selected patients (10). However, postoperative PCNL in most hospitals still requires hospitalized observation, and day-surgery PCNL has not been widely carried out. UMP is an improvement based on PCNL. Compared with PCNL, UMP had the following advantages: smaller channel, visualization by puncture, more possibility of tubeless. The advantages of the device, the establishment of multi-disciplinary day surgery procedures and surgeons with extensive PCNL surgery experience. All these factors putting together allowed us to successfully complete 358 day-surgery semi tubeless UMP and conduct a retrospective analysis.

It should be noted that the size of the stones is not the most important factor in choosing whether to perform UMP surgery. Desai et al. proved that compared with other surgical methods, UMP showed a higher safety for not only $\leq 2 \mathrm{~cm}$ stones but also $>2 \mathrm{~cm}$ stones; and it might bring a lower bleeding risk compared with PCNL and mini-PCNL $(6,11)$; meanwhile, it was superior to ESWL and RIRS in the stone clearance rate without an increasing of the surgical complications (6). In this retrospective study, although there were 29 patients with staghorn stones, the vast majority did not exceed $2.5 \mathrm{~cm}$. The longest diameter was $3.7 \mathrm{~cm}$.

First looking at the safety of day-surgery semi tubeless UMP. In this study, the complication rate was $7.82 \%$ and Clavien I and II accounted for $89.29 \%$. It has been reported that the incidence of UMP-related complications was $5 \%$, and most of them were at the low-level 


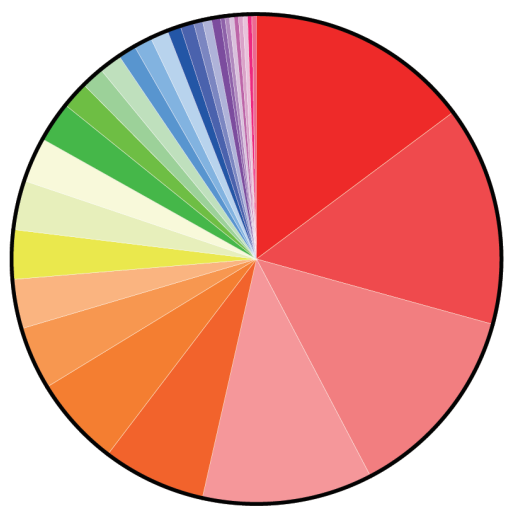

Total: 338

$\mathrm{CaOxm}=$ calcium oxalate monohydrate $\mathrm{CaOxd}=$ calcium oxalate dihydrate CarA $=$ Carbonate apatite Hyd =hydrocyapatite AAU =ammonium acid urate HMAP =hexahydrate magnesium ammonium phosphate CahPd $=$ Calcium hydrogen phosphate dihydrate UASS $=$ Uric acid sodium salt

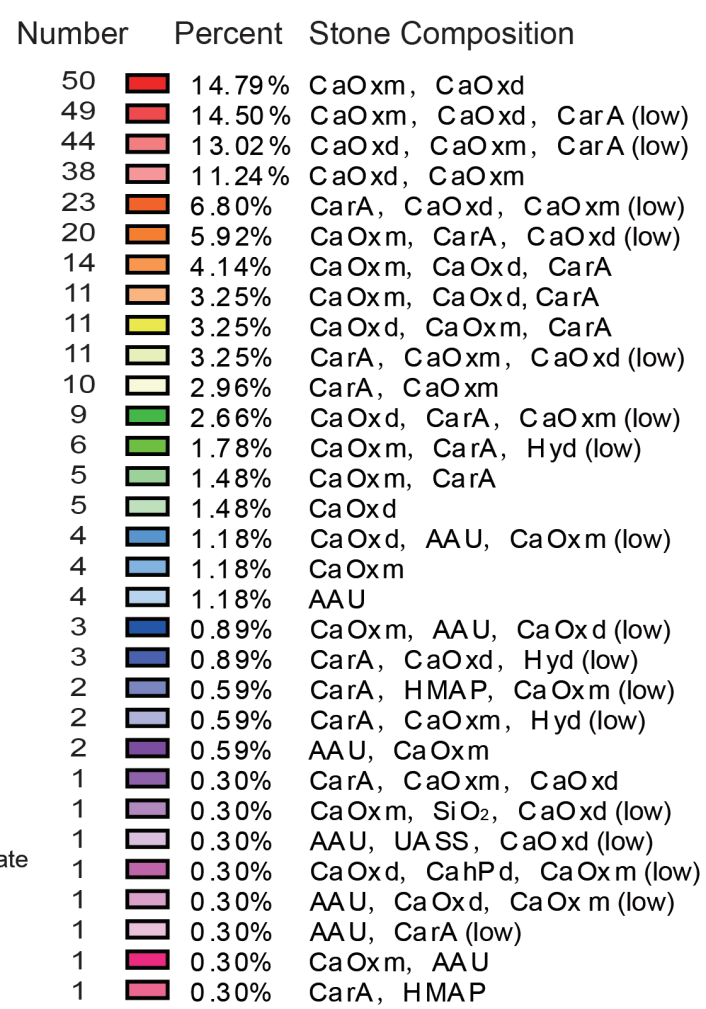

Figure 1 The results of stone analysis.

region (11). Meanwhile, their incidence was significantly lower than that of mini-PCNL (26.9\%) (12) and PCNL (15.6\%) (8). In these literatures about the complications of UMP surgery, patients did not adopt the mode of daysurgery or tubeless, but the complication rate was similar to the results of our study. So, short hospitalization time and semi-tube management were not elements which obviously affected the complications of UMP surgery.

Desai et al. thought that the cause for low incidence of UMP-related complications was ascribed to fine channel (11). A smaller channel brings a lower degree of dilation. But the authors believed that the reason was more than that. For most Chinese urologists, the puncture has been performed under ultrasound guidance. The UMP nephroscope with a diameter of $1 \mathrm{~mm}$ can pass through the outer sheath of the puncture needle to determine whether the puncture position is accurate before dilation. We called it visualization of UMP. Therefore, the precise puncture brought by visualization of UMP may be the main reason for the reduction of complications, not just fine channels.

In this retrospective study, three patients got renal embolized because of arterial bleedings, and 10 cases were shown to have perirenal hematoma by the postoperative image, which was considered to be caused by the channel puncturing and excessive movement of the metal sheath in operation. The renal embolization rate was $0.84 \%$. The rate of embolization was also lower than PCNL surgery. Nephrostomy tubes have been placed by 6 persons. Two with caliceal diverticulum stones and 4 cases of diabetics with long operation time ( $>45 \mathrm{~min})$. Based on the data from it, the authors believe that the UMP-induced postoperative renal bleeding is rare and the nephrostomy tube has no obvious effect on postoperative hemostasis from the view of oppression in bleeding point of the channel. Compared with the previous about 3 days of hospitalization after surgery, discharge after 24 hours may cause increased patient activity, but not bleeding rate. In fact, in order to prevent the channel bleeding, the following measures have been taken in our hospital: after completing the surgery, the sheath was withdrawn under direct vision while observing whether bleeding occurs; if there was bleeding, hemostasis should be performed using the holmium laser.

No urinary-derived sepsis was noted after surgery in this study and no one was readmitted because of infection. Ten patients developed fever after surgery (maximal $38.7^{\circ} \mathrm{C}$ ) and got improved after upgrade of antibiotics. The 
cause was considered to be infection, other possible factors such as absorption fever could not be excluded. Overall, the low incidence of infection in UMP was believed to be related to the following points: (I) preoperative selection and preparation of patients. As a day-surgery, the patient received a routine examination for infection indicators before admission. In case of infection, the patient should be given active anti-infection treatments and it is highly likely that patients will no longer be included in the day-surgery. Before operation, the patients should not exhibit the signs of infection on blood biochemical examination and fever, and should have urinary $\mathrm{WBC}<5 / \mathrm{HP}$ on microscopy. (II) Short operative time. The average operative time was $29.64 \mathrm{~min}$, which is attributed to two aspects. One was due to the extensive surgical experience, and the other was the size of the stone. (III) Intraoperative and postoperative use of antibiotics. Antibiotics were not only used during the surgery in the prophylactic dose, but also were given routinely once after surgery.

Infections, especially serious ones, have always been needed to be avoided by surgeons. It's like the sword of Damocles and always needs to be kept in mind. DJ tubes were placed in 11 patients and nephrostomy tubes were 6 . The reason of placement was to avoid obstructions caused by various conditions. such as PUJ stenosis, large blood clots in the renal pelvis, etc. Diabetics are susceptible to infection, especially if the operation time is kind of long. Therefore, there were 9 patients with long operation time (>45 min), who were not tubeless. The other UMP-related complications (e.g., pleura, lung and intestine injury, etc.) were rare in literatures, and were not also observed in this study.

PCNL has a high stone clearance rate, but the risk of postoperative bleeding also increases (13). When RIRS is adopted for treatment of kidney stones, the stone clearance rate is far below the anterograde surgery, regardless of use of ureteroscope sheath. The highest stone clearance rate of RIRS was $84.9 \%$, as reported in 2015 (14). A prospective cohort study conducted by Datta $e t a l$. adopted the size of stone $>2 \mathrm{~mm}$ as the criteria for residual stones and proposed that the stone clearance rate was $74 \%$ one day after UMP and $81 \%$ one month later (15). This retrospective study showed that the stone clearance rate was $91.62 \%$ one day after surgery, which was higher than that in literatures. The reasons might be associated with the factors below: (I) this study took $>3 \mathrm{~mm}$ as the criteria for residual stones while some studies used $>2 \mathrm{~mm}$. (II) When UMP is used, selection of the patients is stricter taking into account the specificity of day surgery, although there was no clear limit in the size and number. UMP will not be performed in the patients with more complex structure of renal calyx and pelvis, lower possibility of one-time complete clearance based on preoperative considerations or difficulty in processing multiple stones using a single channel. (III) Operator' experience: the surgeons carrying out UMP in our hospital were all the specialists in treating stones. They gained rich experience during engaging in PCNL for a long time, and each had the cumulative amount of PCNL up to 1,000 or more.

In this retrospective study, 326 (91.06\%) patients which received overnight observation were discharged within 24 hours from admission. This ratio was satisfactory. This success, however, may not translate to all surgeons, hospitals, or patients. When evaluating whether to implement a day-surgery program, a practice should consider a number of important factors including the team performing the procedure, the patient, and the hospital (16). By summarizing this retrospective study about the day-surgery semi tubeless UMP, we proposed the following experience: (I) the size of the stone is not the decisive factor, $2 \mathrm{~cm}$ can only be used as a reference; (II) by referring to radiographic assessment before surgery, those in whom all stones could be removed once using a single channel were chosen; (III) the patients don't have the infection or the infection has been effectively controlled before surgery; (IV) the patients don't have a single functional or anatomical kidney; (V) the patients don't suffer from serious systemic diseases, and are believed to be discharged within $24 \mathrm{~h}$ based on the general condition and tolerance by the doctors of the anesthesia department; (VI) the surgeon must have the necessary skills and employ the use of all valuable tools to facilitate the procedure.

This study is a retrospective non-controlled study, and its results may be partially biased. We will further conduct prospective controlled studies based on the results of this study.

Our hospital is the largest tertiary medical center in eastern China. All patients this time came from 22 different provinces and cities in China. Therefore, the results of this study have generalizability for Chinese people.

\section{Conclusions}

For day-surgery semi tubeless UMP, experienced surgeons 
gain excellent patient outcomes in appropriately selected patients. Day-surgery semi tubeless UMP is worth promoting.

\section{Acknowledgments}

Funding: Clinical promotion of ultra-mini percutaneous nephrolithotomy (research project of Shenkang Hospital Development Center 16CR4006A).

\section{Footnote}

Reporting Checklist: The authors have completed the STROBE reporting checklist. Available at http://dx.doi. org/10.21037/tau-20-1060

Peer Review File: Available at http://dx.doi.org/10.21037/ tau-20-1060

Conflicts of Interest: All authors have completed the ICMJE uniform disclosure form (available at http://dx.doi. org/10.21037/tau-20-1060). The authors have no other conflicts of interest to declare.

Ethical Statement: The authors are accountable for all aspects of the work in ensuring that questions related to the accuracy or integrity of any part of the work are appropriately investigated and resolved. All procedures performed in this study were in accordance with the Declaration of Helsinki (as revised in 2013). This study was reviewed and approved by Ethics Committee of Renji Hospital Affiliated to Shanghai Jiao Tong University School of Medicine (KY2020-058). All patients enrolled completed the informed consent form.

Open Access Statement: This is an Open Access article distributed in accordance with the Creative Commons Attribution-NonCommercial-NoDerivs 4.0 International License (CC BY-NC-ND 4.0), which permits the noncommercial replication and distribution of the article with the strict proviso that no changes or edits are made and the original work is properly cited (including links to both the formal publication through the relevant DOI and the license). See: https://creativecommons.org/licenses/by-nc-nd/4.0/.

\section{References}

1. Romero V, Akpinar H, Assimos DG. Kidney stones: a global picture of prevalence, incidence, and associated risk factors. Rev Urol 2010;12:e86-96.

2. Fernström I, Johansson B. Percutaneous pyelolithotomy. A new extraction technique. Scand J Urol Nephrol 1976;10:257-9.

3. Kukreja R, Desai M, Patel S, et al. Factors affecting blood loss during percutaneous nephrolithotomy: prospective study. J Endourol 2004;18:715-22.

4. Yamaguchi A, Skolarikos A, Buchholz NP, et al. Operating times and bleeding complications in percutaneous nephrolithotomy: a comparison of tract dilation methods in 5,537 patients in the Clinical Research Office of the Endourological Society Percutaneous Nephrolithotomy Global Study. J Endourol 2011;25:933-9.

5. Mishra S, Sharma R, Garg C, et al. Prospective comparative study of miniperc and standard PNL for treatment of 1 to $2 \mathrm{~cm}$ size renal stone. BJU Int 2011;108:896-9; discussion 899-900.

6. Desai J, Zeng G, Zhao Z, et al. A novel technique of ultramini-percutaneous nephrolithotomy: introduction and an initial experience for treatment of upper urinary calculi less than $2 \mathrm{~cm}$. Biomed Res Int 2013;2013:490793.

7. Jones P, Bennett G, Dosis A, et al. Safety and Efficacy of Day-case Percutaneous Nephrolithotomy: A Systematic Review from European Society of Uro-technology. Eur Urol Focus 2019;5:1127-34.

8. de la Rosette J, Assimos D, Desai M, et al. The Clinical Research Office of the Endourological Society Percutaneous Nephrolithotomy Global Study: indications, complications, and outcomes in 5803 patients. J Endourol 2011;25:11-7.

9. Schoenfeld D, Zhou T, Stern JM. Outcomes for Patients Undergoing Ambulatory Percutaneous Nephrolithotomy. J Endourol 2019;33:189-93.

10. Singh I, Kumar A, Kumar P. "Ambulatory PCNL" (tubeless PCNL under regional anesthesia) -- a preliminary report of 10 cases. Int Urol Nephrol 2005;37:35-7.

11. Desai J, Solanki R. Ultra-mini percutaneous nephrolithotomy (UMP): one more armamentarium. BJU Int 2013;112:1046-9.

12. Abdelhafez MF, Amend B, Bedke J, et al. Minimally invasive percutaneous nephrolithotomy: a comparative study of the management of small and large renal stones. Urology 2013;81:241-5.

13. De S, Autorino R, Kim FJ, et al. Percutaneous nephrolithotomy versus retrograde intrarenal surgery: a systematic review and meta-analysis. Eur Urol 2015;67:125-37. 
14. Traxer O, Wendt-Nordahl G, Sodha H, et al. Differences in renal stone treatment and outcomes for patients treated either with or without the support of a ureteral access sheath: The Clinical Research Office of the Endourological Society Ureteroscopy Global Study. World J Urol 2015;33:2137-44.

Cite this article as: Chen Q, Cao Y, Xia L, Zhong H, Du Z, Xuan H, Lu M. The retrospective experience of day-surgery semi tubeless ultra-mini percutaneous nephrolithotomy. Transl Androl Urol 2021;10(2):654-661. doi: 10.21037/tau-20-1060
15. Datta SN, Solanki R, Desai J. Prospective Outcomes of Ultra Mini Percutaneous Nephrolithotomy: A Consecutive Cohort Study. J Urol 2016;195:741-6.

16. Wu X, Zhao Z, Sun H, et al. Day-surgery percutaneous nephrolithotomy: a high-volume center retrospective experience. World J Urol 2020;38:1323-8. 